\title{
A Mechanism of Rat Vibrissal Movement Based on Actual Morphology of the Intrinsic Muscle Using Three-Dimensional Reconstruction
}

\author{
Jeong-Nam Kim Ja-Young Yoo Ju-Young Lee Ki-Seok Koh Wu-Chul Song \\ Department of Anatomy, Research Institute of Medical Science, Konkuk University School of Medicine, \\ Seoul, Republic of Korea
}

\section{Key Words}

Arrector pili muscle $\cdot$ Mustache $\cdot$ Vibrissa $\cdot$ Vibrissal

capsular muscle $\cdot$ Rat

\begin{abstract}
The vibrissal capsular muscle (VCM) of the rat is known to differ from the arrector pili muscle. The purpose of the present study was to characterize the rat VCM morphologically using three-dimensional reconstruction. The rat snout skin was fixed, processed with routine histological methods, sectioned serially at a thickness of $10 \mu \mathrm{m}$, and then stained with Masson's trichrome. The sectioned images were reconstructed three-dimensionally using 'Reconstruct' software. The findings confirmed that the VCM is a skeletal muscle attached to the vibrissal follicle such that the latter is rooted within the former. The VCM encircles the follicle almost entirely, from base to apex, and hooks around the follicle caudally. Each one of these capsular muscles is connected to two adjacent follicles in the same row. They overlap each other in the lower part, as the rostral follicular muscle that surrounds the caudal follicle. The present findings suggest that the vibrissae are able to move more freely (under voluntary control) than other general arrector pili muscles, in line with their sensory function.

Copyright ๑ 2012 S. Karger AG, Basel
\end{abstract}

\section{Introduction}

It is known that the morphology and origin differ between the vibrissal capsular muscle (VCM) and the arrector pili muscle. The arrector pili muscle of body hair is smooth muscle and is connected from the dermal papillary layer to the hair follicle. In the rat, the VCM is a blended muscle formed by fibers from the nasolabialis and maxillolabialis muscles [Dörfl, 1982; Wineski, 1985]. They are associated with vibrissal follicles and are only partially inserted into the upper dermis and epidermis. The VCM forms a hook or sling that connects with other vibrissae, and is located in the adipose tissue, allowing the free movement of the follicle [Dörfl, 1982; Wineski, 1985; Haidarliu et al., 2010]. The movement of the vibrissae is controlled by the facial motor nerve [Miyashita and Mori, 1995].

\begin{tabular}{ll} 
Abbreviations used in this paper \\
\hline $3-D$ & three-dimensional \\
TVM & tractor vibrissal muscle \\
VCM & vibrissal capsular muscle
\end{tabular}


Fig. 1. Transverse histologic section of the vibrissal capsular muscle. a The superior region (ring sinus level). The muscle is associated solely with the vibrissal follicles, without any bony attachment. b The middle region. c The inferior region (cavernous sinus level). C2-C5 = Topographic vibrissal arrangement [Haidarliu and Ahissar, 1997]. Scale bars $=500 \mu \mathrm{m}$.

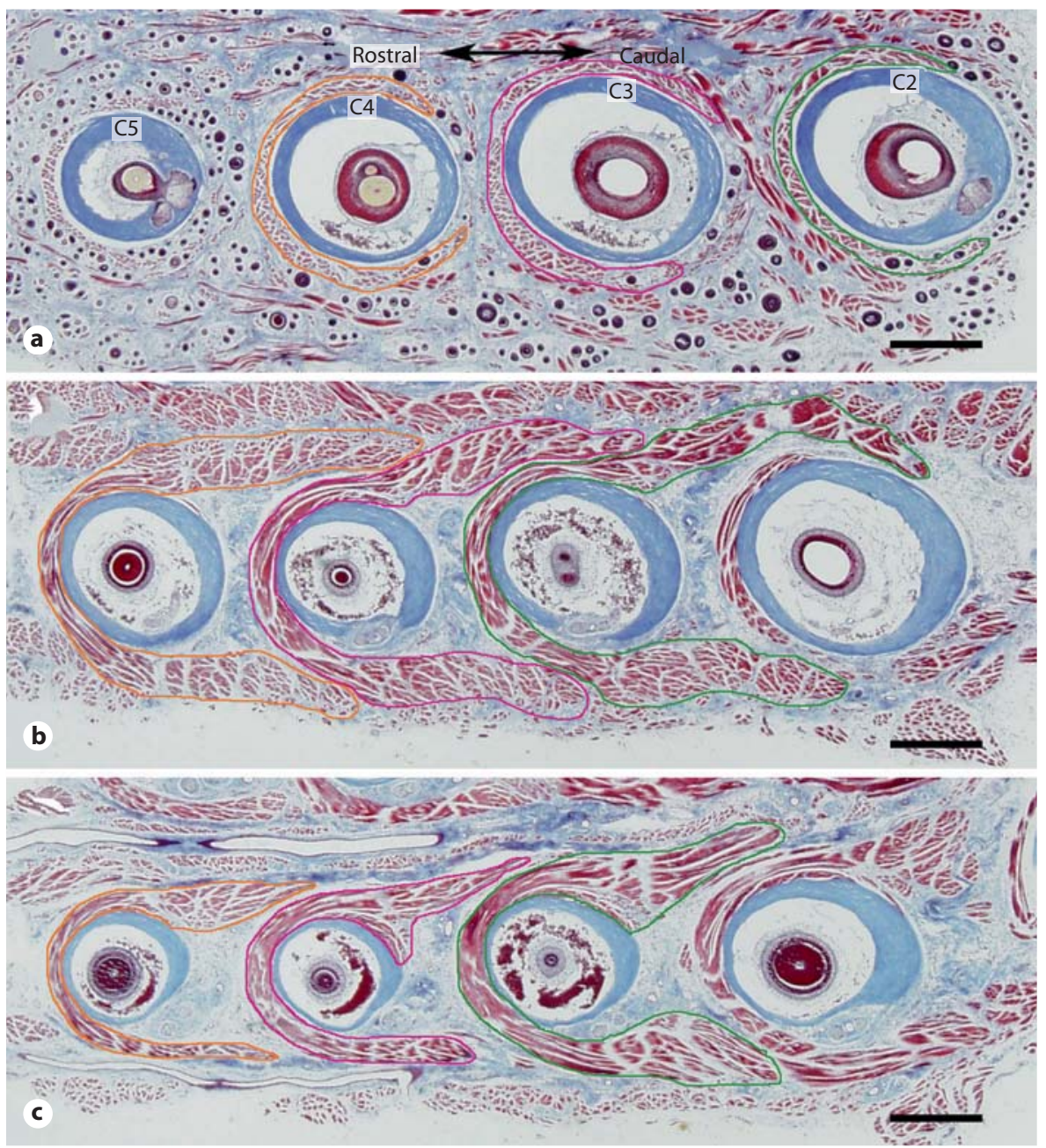

The study by Haidarliu et al. [2010] gave very detailed information about the muscle architecture in the rat vibrissa. However, this study focused mainly on the extrinsic muscle. The present study focused on the morphological aspect of the intrinsic muscle, i.e. the VCM. The two-dimensional schematic drawings obtained in previous studies have not revealed a definitive location or distribution of the VCM.

Cross-sectional studies were shown to be the best method of investigating hair follicles [Headington, 1984], with three-dimensional (3-D) reconstruction based on serial cross sections, which reveal the actual microstructure and function of hair follicles and their appendages [Song et al., 2005, 2006, 2007]. Moreover, our previous 3 -D reconstruction study showed that the structure of the vibrissal follicle-sinus complex differed from previous reports [Kim et al., 2011]. The purpose of the present study was to identify the rat VCM morphologically using 3-D reconstruction. The functional aspects of the VCM were then reevaluated based on morphology.

\section{Materials and Methods}

Materials and Sampling

Sprague-Dawley rats at $10-12$ weeks of age were used. The animals were perfused with $150 \mathrm{ml}$ of saline followed by $600 \mathrm{ml}$ of fixative containing $4 \%$ paraformaldehyde in phosphate-buffered saline. The skin of the lateral region, the harvested region, was C2-C5 and D2-D5 of the vibrissal arrangement of the rat [Haidarliu and Ahissar, 1997], including the vibrissal follicle-sinus complexes. The samples were postfixed with $10 \%$ neutral-buffered formalin. The specimens were trimmed to $15 \times 10 \mathrm{~mm}$ to make them easier to handle and to facilitate the acquisition of images during fixation. 
Fig. 2. Reconstructed vibrissal follicle and VCM. a Superior view. The muscles have the form of a sling surrounding the upper part of the caudal follicle. $\mathbf{b}$ Basal view. The muscles join adjacent follicles of a single row and are attached to rostral and caudal follicles, forming a hook shape. c Lateral view. Each vibrissal follicle is embraced by overlapping muscle. d Posterior and transparent view. The distribution form of the single muscle. It is suggested that the VCM enables free movement of the vibrissal follicles.
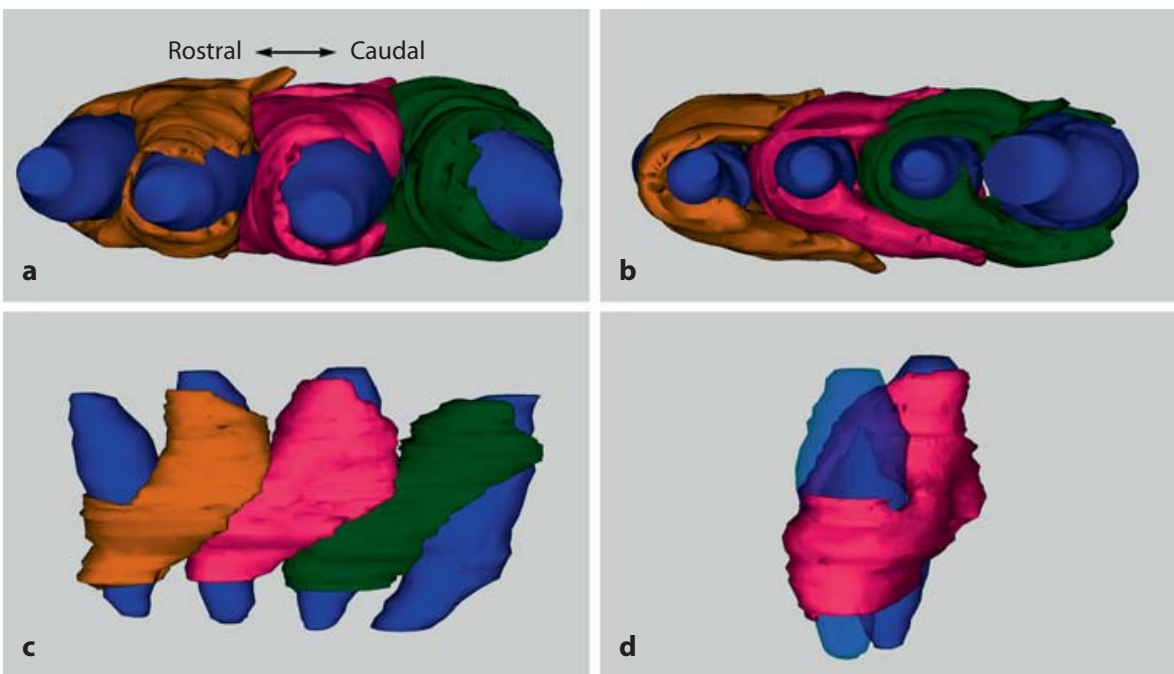

d

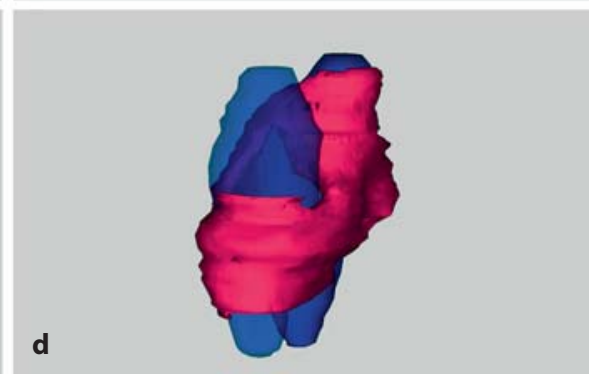

Tissue Processing

After dehydration and paraffin infiltration, each sample was embedded epidermis side down. The paraffin-embedded specimens were cut transversely and serially from the surface of the epidermis down to the lower dermis - to include the full length of the follicles - at a thickness of $10 \mu \mathrm{m}$, and used for 3-D reconstruction. The number of sections varied with the length of the follicles, but ranged from 300 to 400 . The serially sectioned tissues were stained with Masson trichrome.

\section{3-D Reconstruction}

All of the stained sections were photographed using a 2,048 $\times$ 1,536 pixel digital CCD camera (DP70; Olympus, Tokyo, Japan). Three or four vibrissal follicle-sinus complexes were reconstructed from each specimen. 'Reconstruct' software was used to produce the 3-D images of the VCM and the vibrissal follicle (this software was downloaded from http://synapses.clm.utexas.edu/ tools/reconstruct/reconstruct.stm) [Fiala, 2005]. The images were aligned manually using the software. The structures were aligned along the external margin and then reconstructed.

\section{Results}

The VCM surrounding the vibrissal follicle from base to apex was grouped with a rostral follicle and neighbored a caudal follicle in the same row (fig. 1, 2). A single muscle was hook-shaped, encircling the superior region of the caudal follicle and the inferior region of the rostral follicle (fig. 2). The upper muscle fiber of the VCM (ring sinus level) was C-shaped and kept a tight grip on the superior rostral surface of the follicle, with the exception of the superior caudal part of the follicle (fig. 1). The low-

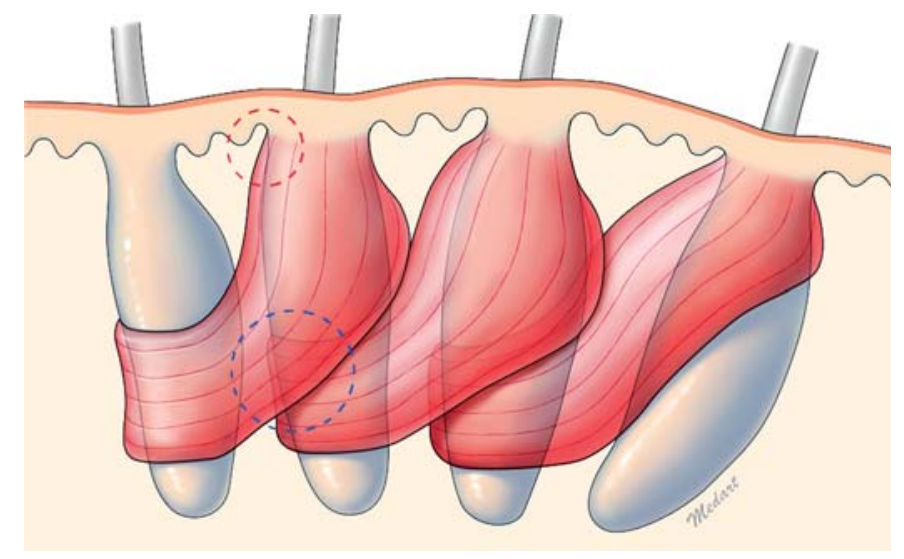

Fig. 3. Schematic drawing of the tractor vibrissal muscle. The muscle fibers in the lower region run approximately parallel with the skin surface, but they run vertically in the upper region. Red circle $=$ Axis of movement of the tractor vibrissal muscle; blue circle $=$ overlapping region .

er fiber of the VCM (cavernous sinus level) was slingshaped and attached to both the rostral and caudal follicles (fig. 2b, c). Individual muscles overlapped each other so that the caudal part of the rostral VCM wrapped partially around the rostral part of the caudal VCM (fig. 2, 3). The direction of the muscle fiber was approximately parallel to the skin surface at the rostral surface of the lower part of the VCM, while it was oblique in the middle parts and vertical in the upper part of the VCM (fig. 3). 


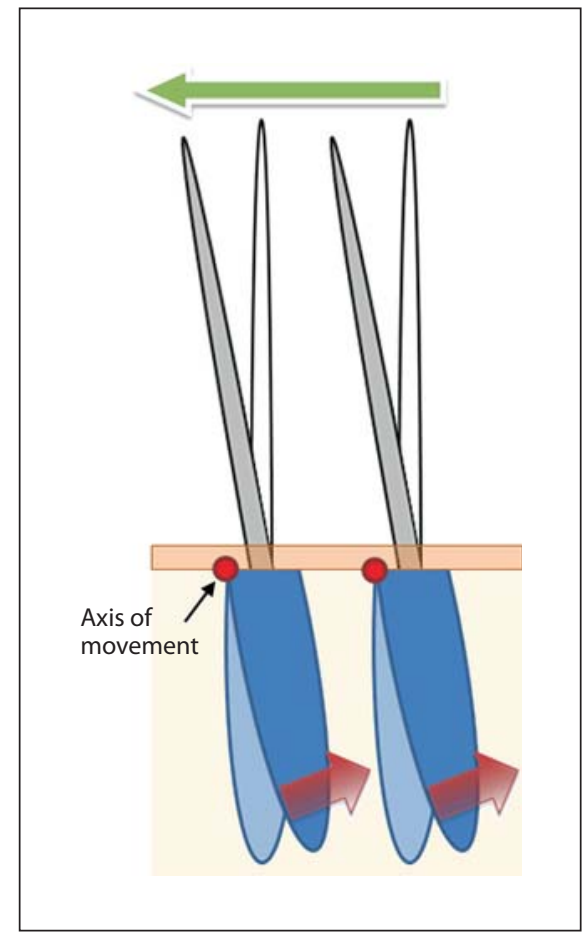

Fig. 4. Schematic diagram of the axis of movement. When the tractor vibrissal muscle contracts, the follicle is pulled in the posterosuperior direction (red arrows) and the vibrissal shaft moves in the anterior direction along the axis of movement. Blue $=\mathrm{Vi}$ brissal hair follicle; red dots = axis of movement of the tractor vibrissal muscle.

\section{Discussion}

The vibrissal follicle is an exceptionally well-innervated, important tactile organ that is present in most mammals [Ebara et al., 2002]. The precise morphology of the rat vibrissal follicle-sinus system has been elucidated previously using 3-D reconstruction [Kim et al., 2011]. The rat vibrissal follicle is a spindle-shaped structure that is embedded within a blood sinus and enveloped within a thick collagenous capsule. Movement of the vibrissae is very important for the detection of environmental information.

The vibrissal follicle was found to be surrounded by skeletal muscle, which comprises both extrinsic and intrinsic fibers. The extrinsic muscles are attached to bones and belong to the group of facial muscles that move the superior lip and nose (the levator labii superioris, maxillolabialis, transverse nasi, and nasalis muscles). The intrinsic muscle fibers make up the VCM, whose function is the erection and protraction of the vibrissal shaft
[Dörfl, 1982; Wineski, 1985; Haidarliu et al., 2010]. Other reports on vibrissal movement and sensation [Berg et al., 2003; Hill et al., 2008] still refer to the earlier two-dimensional schematic drawings [Dörfl, 1982; Wineski, 1985], which limits the ability to explain the functional aspects of the vibrissae based on the known morphology.

The present study has established that the VCM is not a simple band of muscle that tightly surrounds the vibrissal follicle. The single muscle is attached to rostral and caudal follicles (fig. 2d). Furthermore, every muscle overlaps with another in the inferior region of the follicle (i.e., at the cavernous sinus level; fig. 3). This structural relationship can explain the detailed movement of the vibrissae coupled to the response of tactile sense.

We suggest a new model of the vibrissal muscle based on the actual 3-D structure (fig. 3, 4). The vibrissal follicle moves in the posterosuperior direction when the VCM contracts, thus moving the vibrissal shaft in an anterior direction. This concept does not differ from that described by Wineski [1985]. However, we also found that the VCM covered the superior rostral surface of the caudal follicle, and not the superior caudal surface (fig. 2a). This finding is significant since the superior rostral surface may be tightened during VCM contraction, and the superior rostral surface of the follicle would no longer be pushed anteriorly, in spite of the posterosuperior movement of the lower part of the follicle. In other words, this part is fixed and can act as an axis during movement. Furthermore, because this part acts as an axis, it also prevents the overlying skin from being pushed anteriorly. If the skin was pushed anteriorly during VCM contraction, it may influence the movement of the surrounding follicles by contracting the VCM in a similar direction. This would result in the individual vibrissae not functioning independently. However, as mentioned above, the actual VCM appears to cover the rostral superior part of the caudal follicle, thus forming an axis. The superior part does not act on follicular movement directly, but as an axis during movement. Therefore, the superficial skin cannot be pushed anteriorly during movement, and the vibrissae can thus move independently of each other. The individual independent movement of the vibrissae enables a fine distinction of the environment in any situation. A previous study concluded that the axis of movement (motor plant) was displayed in the center of the follicle [Haidarliu et al., 2010]. However, our study found that the axis of movement was displayed at the rostral top of the follicle which provides more stability for movement than the center (fig. 4). It also reduces the movement of skin and senses details. 
The term 'VCM' is a morphological one; however, the most important function of the vibrissae is movement for sensory detection. Therefore, we propose the new term 'tractor vibrissal muscle (musculi tractor vibrissae)' (TVM) to replace VCM, which we consider more precisely describes the characteristic function of the muscle, and is therefore easy to understand.

Based on the morphology of the TVM, the relationship between it and the underlying facial expression muscle was difficult to investigate, other than to say that they are both skeletal muscles. Although previous reports [Dörfl, 1982; Wineski, 1985, Kim et al., 2011] have insisted that the TVM originates from the underlying facial muscle, we suggest that the TVM is an independent muscle whose function is vibrissal movement, and that it is separated from the underlying facial muscle. With the exception of the type of muscle fiber, the TVM is very similar to the arrector pili muscle morphologically and functionally.

\section{Acknowledgements}

This work was supported by a grant from the Korea Science and Engineering Foundation (KOSEF) funded by the Korean government (MEST) (No. R01-2008-000-10189-0). We thank Dr. Kwan-Hyun Youn for the wonderful illustrations.

\section{References}

Dörfl, J. (1982) The musculature of the mystacial vibrissae of the white mouse. J Anat 135: 147154.

Ebara, S., K. Kumamoto, T. Matsuura, J.E. Mazurkiewicz, F.L. Rice (2002) Similarities and differences in the innervation of mystacial vibrissal follicle-sinus complexes in the rat and cat: a confocal microscopic study. J Comp Neurol 449: 103-119.

Fiala, J.C. (2005) Reconstruct: a free editor for serial section microscopy. J Microsc 218: 52 61.

-Haidarliu, S., E. Ahissar (1997) Spatial organization of facial vibrissae and cortical barrels in the guinea pig and golden hamster. J Comp Neurol 385: 515-527.

-Haidarliu, S., E. Simony, D. Golomb, E. Ahissar (2010) Muscle architecture in the mystacial pad of the rat. Anat Rec (Hoboken) 293: 1192-1206.
Headington, J.T. (1984) Transverse microscopic anatomy of the human scalp. A basis for a morphometric approach to disorders of the hair follicle. Arch Dermatol 120: 449-456.

Hill, D.N., R. Bermejo, H.P. Zeigler, D. Kleinfeld (2008) Biomechanics of the vibrissa motor plant in rat: rhythmic whisking consists of triphasic neuromuscular activity. J Neurosci 28: 3438-3455.

Kim, J.N., K.S. Koh, E. Lee, S.C. Park, W.C. Song (2011) The morphology of the rat vibrissal follicle-sinus complex revealed by three-dimensional computer-aided reconstruction. Cells Tissues Organs 193: 207-214.

Miyashita, E., S. Mori (1995) The superior colliculus relays signals descending from the vibrissal motor cortex to the facial nerve nucleus in the rat. Neurosci Lett 195: 69-71.
Song, W.C., K.S. Hu, H.J. Kim, K.S. Koh (2007) A study of the secretion mechanism of the sebaceous gland using three-dimensional reconstruction to examine the morphological relationship between the sebaceous gland and the arrector pili muscle in the follicular unit. Br J Dermatol 157: 325-330.

Song, W.C., K.S. Hu, K.S. Koh (2005) Multiunit arrector pili muscular structure as a variation observed by using computer-based three-dimensional reconstruction. Cell Tissue Res 322: 335-337.

Song, W.C., W.J. Hwang, C. Shin, K.S. Koh (2006) A new model for the morphology of the arrector pili muscle in the follicular unit based on three-dimensional reconstruction. J Anat 208: 643-648.

Wineski, L.E. (1985) Facial morphology and vibrissal movement in the golden hamster. J Morphol 183: 199-217. 